\title{
Quelques aspects de comite de Paix et de Sécurité de l’Union Africaine
}

\begin{abstract}
Résumé:
L'Union Africaine, née à Durban (Afrique du sud), en 2002, s'est dotée d'un ambitieux organe baptisé "Conseil de Paix et de Sécurité ", chargé de la gestion et du règlement des conflits, notamment interétatiques qui rongent l'Afrique comme ceux de la Somalie, du Soudan (Darfour...)

A cet effet, il s'appuie sur un ensemble de structures consistant dans un «Groupe des Sages » de cinq personnalités africaines en tant que consécration d'une veille tradition de l'Organisation de l'Unité Africaine (OUA) de ses comités ad hoc ; un "Système d'alerte rapide »; une « force africaine en attente ; un Etat major »).

Mais cet organe est viscéralement porteur de sa faiblesse et de son incapacité à s'acquitter du mandat à lui assigné : la tendance de certains de ses membres à s'impliquer dans tel conflit pour développer des prétentions à l'hégémonie et/ou à servir d'outil utile aux puissances extra-africaines comme l'Ethiopie en Somalie...les difficultés financières, par " défaut de coopération » des membres de l'Union, aggravent son déficit pour aliéner son indépendance.
\end{abstract}

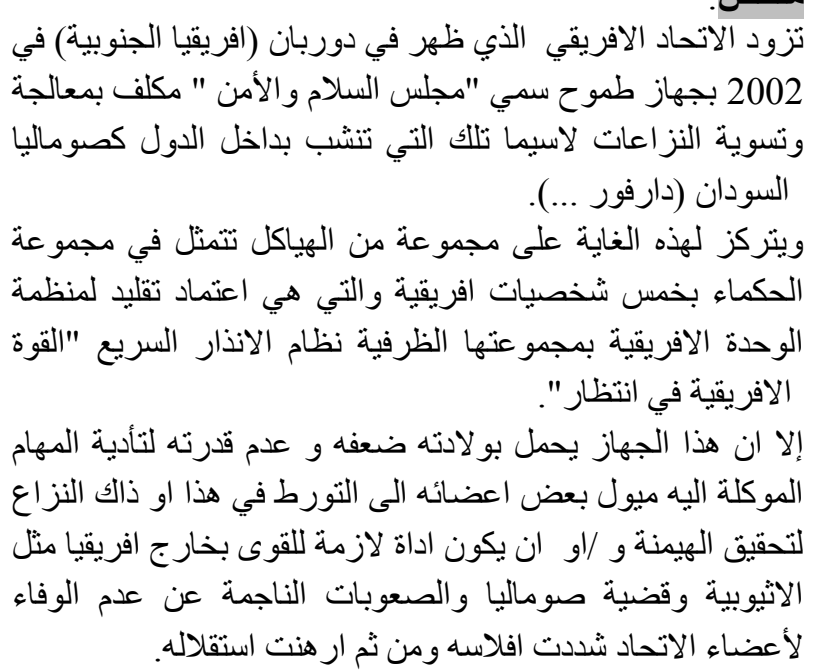

\section{BOUCHERIKHA AMMAR}

Faculté de droit

Université des Frères Mentouri

Constantine

\section{Introduction :}

Par l'acte lui-même de créer une nouvelle organisation, en l'occurrence l'Union africaine, les fondateurs de celle-ci se sont inscrits dans la dynamique de rupture avec le défunt système de l'OUA à la quelle elle a succédé lors du Sommet de Durban (Afrique du Sud) en 2002 d'un Système de Paix et de Sécurité, clef de voûte du développement auquel il est associé.

$\mathrm{Ce}$ système aux relents présidentiels congolais serait de l'avis de M.ROCARD, un système de solidarité interétatique. 
Il puise également dans les précédents africains au titre des forces de maintien de la paix déployées par la Communauté des Etats d'Afrique de l'Ouest (CEDEA) au Liberia victime en 1990 d'une guerre civile.

Mais le mécanisme de l'ONU avec son conseil de sécurité agissant notamment dans le cadre du chapitre VII de la Charte et alors même qu'il est tout à fait le contraire de sa raison d'être n'a pas d'équivalent.

L'Afrique est l'un des continents ravagés par les guerres civiles et les conflits interétatiques, ce qui fait d'elle un consommateur des opérations de maintien la paix.

L'organe central au cœur dudit système est le Conseil de Paix et de Sécurité (CPS) au mandat difficilement réalisable de par le défaut d'unité de ses membres et de finances alors même qu'il s'appuie sur des structures, présentes et à venir viables à l'honneur de l'Union.

I- Le mécanisme de paix et de sécurité :

Le Conseil de Paix et de Sécurité, inspiré du Conseil de Sécurité de l'ONU et par ailleurs Successeur du Mécanisme pour la prévention, la gestion et le règlement des conflits de l'OUA, est l'organe clef représentatif de l'Union africaine en matière de paix et de sécurité ; autant un organe ambitieux et coûteux qui n'est pas à l'abri de la manipulation et du risque de carence à l'instar du Mécanisme considéré à l'efficacité en effet douteuse voire de marginalisation.

\section{équilibré :}

$I_{1}$ - Le Conseil de Paix et de Sécurité : un organisme restreint et

Le conseil de Paix et de Sécurité n'est pas un organe plénier, mais un organe restreint sans doute par souci d'efficacité composé de 15 membres au mandat de trois ans pour l'Algérie (Afrique de l'Ouest), l'Algérie (Afrique du nord), l'Ethiopie (Afrique de l'Est), le Gabon (Afrique Centrale) et l'Afrique du Sud (Afrique Australe); et de deux ans, pour le Ghana, le Sénégal et le Togo (Afrique de l'Ouest), la Libye (Afrique du Nord); le Kenya et le Soudan (Afrique de l'Est), le Cameroun et le Congo (Afrique Centrale), le Lesotho et le Mozambique (Afrique Australe).

La "dualité" ainsi du mandat serait justifiée par la nécessité d'assurer la continuité et le suivi des dossiers et participerait de la discrimination positive entre Etats influents, puissants et riches ou supposés tels et ceux qui le sont moins, mais dans le cadre du respect des principes de rotation et de répartition géographique équitable.

Les principes dont s'agit ont donné lieu à de nombreux débats, certains Etats revendiquant d'autant l'égalité pour chaque sous-région par son attribution de trois sièges qu'elle ne se réduisait pas à l'importance géographique de la région mais à la capacité de contribution; d'autres faisant valoir la pratique traditionnelle de l'organisation fondée sur l'équité régionale par l'octroi de plus de sièges à la sous-région composée du plus grand nombre 
d'Etats de l'Afrique de l'Ouest réclamant quant à eux un statu dérogatoire au principe d'égalité par le bénéfice d'un siège supplémentaire justifié à leurs yeux par la supériorité numérique de la région avec 15 Etats contre 6 Etats pour l'Afrique du Nord (1).

Mais le risque est que le Conseil ne devienne un instrument aux mains d'une poignée d'Etats pour servir leurs intérêts propres et/ou des tiers notamment extra africains par procuration, à l'instar du Conseil de Sécurité des Nations Unies " converti" en filiale su secrétariat d'Etat américain et ...du Pentagone ; cela sans compter les germes "recommandables" aux antipodes de l'Etat de droit, de la démocrate et de la bonne gouvernance pourtant inscrits dans l'acte constitutif de 1'Union africaine en tant qu'objectifs (2). Et/ou à tendance hégémonique; ou de déviation en égard à l'incertitude qui pèse sur ses pouvoirs et attributions et que suggère la complexité des rapports institués entre le Conseil et le président de la commission (3).

Il est assisté -cela vaut également pour le Président de la Commissiond'un "Groupe des sages" -qui rappelle les fameux "Comité des sages" tels que le "Comité des sages" pour le Sahara Occidental- de cinq personnalités africaines, nommées par la Conférence pour trois ans en raison, est-il indiqué, de "leur contribution à la causé de la paix, de la sécurité et de développement" en Afrique; elle sont sélectionnées par le Président de la commission "après consultation des Etats membres concernés"(4).

Ce comité est investi d'une sorte de mission consultative auprès du Conseil -mais aussi de Président de la Commission- relativement aux questions de maintien et de promotion de la paix, de la sécurité et de la stabilité sur le continent.

Il initie les actions qu'il juge appropriées pour prévenir les conflits, tout comme il le fait à la demande du Président de la Commission et par le truchement de ce dernier la Conférence. "Ses modalités de fonctionnement sont élaborées par le Président de la Commission, paradoxalement" présenté comme un organe exécutif des décisions du Conseil, agissant sous son autorité, dont l'organisation et le déploiement des missions d'appui à la paix...et "en consultation avec toutes les parties" ?!

Cela dit, le Conseil s'appuie, en outre, sur un "Système d'alerte rapide" à l'effet de facilité "la prévision et la prévention des conflits" ; il est constitué d'un "centre d'observation et de contrôle" (dit "salle de veille"), chargé de la collecte et de l'analyse des données et "des unités d'observation et de contrôle des mécanismes régionaux"(art.12).

Et en vue d'assurer l'efficacité de son fonctionnement, une collaboration avec l'ONU, ses agences et autres organisations internationales, est prévue, le Président de la commission quant à lui y étant associé au titre de l'élaboration par lui des "détails pratiques" de la mise en place du système. 
Comme son nom l'indique, il a l'aptitude à se déployer en un temps record suivant l'apparition de la crise et qui s'incorporerait dans la diplomatie préventive (5).

Une "Force Africaine pré positionnée" baptisée aussi une "Force Africaine en attente" composée de contingents multidisciplinaires en attente, civils et militaires, stationnés dans leur pays d'origine et appelés à se déployer rapidement, y compris à "intervenir dans un Etat membre dans certaines circonstance", est créée (art.13 c 3 ).

Il s'agit là évidemment d'un grand progrès quand on sait que les Nations Unies elles-mêmes ne disposent d'aucune force, d'aucun fond et d'aucun équipement pour le maintien de la paix (6).

Enfin, pour compléter le dispositif, il est prévu, de mettre en place un état-major permanent chargé de conseiller et d'assister le Conseil de Paix et de Sécurité sur les aspects militaires $\left(\operatorname{art} .13 \mathrm{~g}_{3}\right)$. Mandat en somme modeste en deçà de ce que prévoyant de Document de Denis Sassou N'Guesso et consistant à prévenir les crises et réagir, armé de contingents mobilisables aisément (7).

Autant les structures considérées sont ambitieuses autant la mission à lui impartie.

$I_{2}$ - Le conseil de Paix et de Sécurité : un organe de prévention et de gestion des conflits :

Pour Alpha Oumar Konaré, le Conseil de Paix et de Sécurité est un "organe permanent chargé de la prévention pour la paix et la sécurité, le rétablissement de la sécurité, de la reconstruction après les conflits".

Ceci est conforme, en effet, avec les objectifs de l'Union Africaine au titre de l'article 3 de son acte constitutif.

Fonctionnant sur la base du principe de non-indifférence en rupture avec l'OUA fidèle aux principes de souveraineté et de non-ingérence qui a pourtant justifié les interventions militaires occidentales baptisées cyniquement "interventions humanitaires", le Conseil de Paix et de Sécurité recommande à la Conférence, organe suprême de l'Union, le droit d'intervenir dans les situations de génocide, de crimes de guerre et contre l'humanité ; en somme les critères proposés pour fonder "l'intervention humanitaire".

Le garde-fou de la non-ingérence risquerait de céder quand l'appréciation de telle ou

telle situation butte sur l'opposition d'un de ses membres.

La codification d'un tel principe éminemment politique, donc subversif et déstabilisateur peut légitimer les interventions militaires extra-africaines notamment, outre la France, les Etats-Unis avec leur premier commandement militaire unifié pour l'Afrique (AFRICOM), le $1^{\text {er }}$ octobre 2007, pour, officiellement, aider et prévenir la guerre par le renforcement des capacités militaires africaines et par l'assistance humanitaire. 
Le Conseil de Paix et de Sécurité est investi d'une diplomatie préventive qui est un moyen d'empêcher que les différends ne se transforment en conflit armé (8).

Sa première mission en tant que Force de maintien de la paix durant la guerre dite civile du DARFOUR a consisté à déployer en 2007 environ 7000 hommes. Mais ceux-ci ne sont pas suffisants au regard de l'ampleur de la tâche. Aussi l'Union a réclamé l'augmentation du soutien financier des missions de la paix au DARFOUR, reprochant à l'ONU de faillir à ses propres engagements de financement et de concours technique (9).

II- L' handicap du conseil de Paix et de Sécurité :

Le Conseil de Paix et de Sécurité connaît un double handicap tenant à sa composition hétéroclite et au manque de finances, qui aliénerait son efficacité.

II $_{1^{-}} \quad$ Le Conseil de Paix et de Sécurité : un organe miné par ses contradictions internes sur lesquelles se greffe l'interférence étrangère :

Le Conseil de Paix et de Sécurité comporte en son sein des Etats concernés et intéressés, situation qui fait d'eux "juge et partie"- par leur implication directe ou indirecte militaire ou autre, aux côtés de l'une ou l'autre partie au conflit ; ou eux-mêmes victimes de tendances centrifuges suscitée et assistées dans le but d'affaiblir l'Etat en cause. Ainsi, l'Ethiopie qui abrite le siège de l'Union, en sus de sa qualité de membre dudit organe, n'a pas hésité à intervenir militairement comme sous-traitant des Etats-Unis en été 2007 en Somalie pour officiellement parer au "péril islamiste" que représenteraient les Tribunaux islamiques, alors que ses ambitions régionales ne sont pas à démontrer depuis la guerre de l'Ogaden. Récemment, la République démocratique du Congo pour venir à bout de la nouvelle rebellion soutenue vraisemblablement par le Rwanda est "assistée" par les troupes angolaises.

Certes, leur action pourrait trouver une base légale dans l'article $3_{\mathrm{h}}$ de l'Acte Constitutif de l'Union africaine et l'article $4_{h}$ du Protocole relatif à la création du Conseil de Paix et de Sécurité, mais ce droit à l'intervention sollicitée s'applique à l'Union et non pas à des Etats agissant individuellement ou collectivement.

Le Nigeria lui-même était guidé dans son intervention avec d'autres Etats africains au Liberia par les prétentions à l'hégémonie.

Quant à la Lybie, son profil subversif et à tendance hégémonique ne saurait mieux s'exprimer ici dans l'aide fournie à certains Etats et là dans son projet, qui tiens d'une vue de l'esprit, des Etats-Unis d'Afrique.

D'autres Etats à l'image du Tchad ne se résignent pas à s'affranchir, au nom du maintien au pouvoir du régime, de la tutelle de l'ancienne puissance coloniale pour "neutraliser" en effet la rebellion, un succédané à l'alternance.

Par ailleurs, la présence au cours de la réunion de l'Organe central des parties au conflit, et même leur participation au débat est contre-productive en 
tant qu'elle a pour effet de faire éluder les questions dites fâcheuses d'autant que ces parties brandissent l'étendard de la non-ingérence.

C'est dire que la capacité du Conseil de Paix et de Sécurité à s'acquitter des missions à lui dévolues réside dans l'élaboration et la mise en œuvre d'un projet identitaire commun, cet idéal auquel aspire hélas la minorité et encore!

\section{$\mathbf{I I}_{2}-$ L'handicape financier :}

Il est créé un Fonds Spécial dénommé Fonds de la paix, destiné à financer les missions de maintien de la paix et opérations connexes; il est alimenté par des crédits prélevés sur le budget ordinaire de l'Union Africaine, " y compris les arriérés des contributions, les contributions volontaires"(art.21 $\left.1_{1} \mathrm{et}_{2}\right)$. Les Etats "pourvoyeurs peuvent être invités à prendre en charge le coût de leur participation pendant les trois premiers mois"(art.21). (10).

Or, l'Union africaine souffre cruellement d'un manque d'argent, désigné par l'euphémisme de faiblesse de moyens financiers par le secrétariat général de l'OUA, peu après la création de l'Organe central en 1993, si bien que les puissances extra-africaines inscrivaient leurs contributions financières en fonction de leurs intérêts propres.

Les contributions volontaires publiques ou privées destinées à alimenter les caisses de la "Force africaine en attente" sont hypothétiques.

Certes, un dispositif de sanctions gradué et dissuasif est mis en place contre les membres qui ne versent pas leurs contribution dues, allant de la suspension du droit de l'Etat membre de prendre la parole, voter...lorsque le montant des arriérés s'élève à deux (2) ans; à celle de faire renouveler les contrats d'emploi de \{ses\} nationaux, "bénéficier de fonds de l'Union pour de nouveaux projets"(art.35 de l'Acte constitutif).

Mais l'histoire de l'OUA renseigne de l'inefficacité de telles sanctions auxquelles l'indifférence des uns par défaut d'"appartenance communautaire", ou de "citoyenneté africaine", la dispute à la faillite financière des autres, situation d'autant, préoccupante que la crise financière-née aux Etats-Unis- a fait entrer des pays développés aux économies compétitive, comme l'Allemagne, dans la récession.

L'ONU elle-même à laquelle est réclamée l'augmentation du financement des

missions de paix par exemple au Darfour, a des difficultés financières qui résultent $\mathrm{du}$ fait que les membres ne versent pas ponctuellement et intégralement leurs quotes-parts que la menace ou le recours à l'article 17 de la Charte n'y peut rien, cela indépendamment du spectre de la crise financière mondiale.

En 1993, l'OUA avait créé un Fonds pour la paix, mais n'avait recueilli en l'espace d'une décennie que 96 millions de dollars dont plus des trois quarts 
du montant, provenaient de ses partenaires, contrastant ainsi avec l'Algérie qui avait, quelques années plus tôt versé 10 millions de dollars, au Fonds spécial pour la SWAPO, créé à son initiative, et continue à être l'acteur privilégié en matière de paix et de sécurité comme l'atteste sa médiation réussie dans le conflit Ethiopie-Erythrée.

En tout état de cause, la paix en Afrique continue de dépendre de l'aide extérieure, le principal bailleur de fonds étant l'Union européenne.

Néanmoins l'aide n'est pas aussi désintéressée qu'on le laisse croire ; et les opérations de maintien de la paix sont disputées par des puissances extraafricaines qui s'arroge un "droit de tutelle" sur des pays faibles. Le déploiement par la France de 4000 soldats en Côte d'Ivoire et sa récente intervention aux côtés des troupes gouvernementales Tchadiennes sont à cet égard significatifs.

\section{Conclusion :}

La paix et la sécurité en Afrique sont une chimère aussi longtemps que l'on ne prend pas exemple sur l'Union européenne et que l'on ne renonce pas aux prétentions d'hégémonie et/ou à s'arrimer pas à telle puissance.

La démocratie et la bonne gouvernance, inscrites dans l'Acte constitutif, sont les deux conditions à l'instauration d'une paix durable, c'est ce qui explique le dispositif hostile aux changements anticonstitutionnels, mais inefficace par considération de la pratique mauritanienne récente.

Du reste comment concilier non-ingérence droit d'ingérence -codifié-, "une sorte de boîte de Pandore pour les puissances y compris africaines, et souveraineté, intégrité territoriale". Les difficultés financières qui vont s'aggraver davantage avec la crise financière mondiale sont de nature à saper la paix et la sécurité.

Il n'est pas inutile de citer, à ce propos l'ancien secrétaire général de l'ONU qui déclarait le 10 juillet 2003 à Maputo (Mozambique), à l'occasion du sommet de l'Union africaine: "l'ONU est le reste de la communauté internationale auront beau nommer des envoyés, demander de la paix, tout cela restera vain s'il n'existe pas, ici même, en Afrique, la volonté politique et les capacités nécessaires".

(1) Sur ces débats et leur sanction, voir LECOUTRE (Delphine),"le Conseil de Paix et de Sécurité de l'Union africaine, clef d'une nouvelle architecture de stabilité en Afrique ?" Actualité africaine $\mathrm{n}^{\circ} 212$ ? 2004/4 ? PP. 11 ET 35.

(2) Cf. Kofi ANNANE dans son allocution prononcée le 10 juillet 2003.

(3) Voir supra.

(4) Article $11 \mathrm{du}$ Protocole relatif à la création du Conseil de Paix et de Sécurité de l'Union africaine adopté par la première session ordinaire 
de la conférence de l'Union africaine à Durban (Afrique du sud), le 96 juillet 2002. Désormais il s'écrit Protocole.

(5) Cf. le Rapport annuel sur l'activité de l'Organisation, "Relever les nouveaux défis" de l'ancien secrétaire général de l'ONU, Boutros Boutros Ghali, Nations Unies, New York, 1995, pp. 24 et 55.

(6) Il n'est pas excessif de faire un parallèle avec l'idée de doter les Nations Unies d'une force permanente coercitive "réactualisée" par Boutros Boutros Ghali dans son "Agenda pour la paix". Voir sur ce point, LECLERC-GAGNE (Elise) et LETOURNEAU (Charles), une force permanente pour les Nations Unies ; la paix est-elle possible sans une épée ? Institut d'études internationales de Montréal, Bulletin $n^{\circ} 78$, janvier 2006.

(7) Cf. ROCARD (Michel), un système de solidarité entre Etats ? Géopolitique africaine 2003(Internet).

(8) Rapport annuel op. cit.pp., 241 et 55.

(9) Après de mois de pressions diplomatiques, le Soudan a finalement accepté en juin 2007 le déploiement au Darfour d'une force hybride UA-ONU.

(10)Cf. LECOUTRE (Delphine) op.cit. , p.9).

Bibliographie :

I- $\quad$ Textes (traités et autres)

Charte des Nations Unies, 26 juin 1945.

Charte de l'Organisation de l'Unité africaine, 25 mai 1963.

Acte constitutif de l'Union africaine, 11 juillet 2000.

Protocole relatif à la création du Conseil de Paix et de Sécurité de l'Union africaine, www.peacean.org/uploads/pse-protocolefr.pdf.

Rapport sur le Conseil de Paix et de Sécurité, $19^{\mathrm{e}}$ Sommet de l'Union africaine, www.operations paix.net/.../17-fr-vRapport-sur-le-cps-No-38/pdf.

Rapport annuel 2013, de la Commission européenne, Facilité de soutien à la paix pour l'Afrique, www.europa.en/meetdocs/2014-2019...apf-web-fr.pdf.

II- Doctrine

BOURGI, Albert," L'Union africaine entre les textes et la réalité ",www.diplomatie.gouv.fr/fr/IMG/pdf/Bourgi..pdf.

CHOUALA, Yves Alexandre, Puissance, résolution des conflits et sécurité collective à l'ère de l'Union africaine, www.diplomatie.gouv.fr/fr/IMG/pdf, 19 288-306 pdf.

LECLERC-GAGNE, Elise et LETOURNEAU Charles, "Une force permanente pour les Nations Unies. La paix est-elle 
possible sans épée? Institut d'études internationales de Montréal, Bulletin n ; 78, janvier 2006.

LECOUTRE, Delphine, Le Conseil de paix et de sécurité de l'Union africaine, clef d'une nouvelle architecture de stabilité en Afrique, Actualité africaine, n ; 212, 2004/4, PP. 11-35.

ROCARD, Michel, Un système de solidarité entre Etats? Géopolitique africaine 